\title{
Impacto de los trastornos hipertensivos, la diabetes y la obesidad materna sobre el peso, la edad gestacional al nacer y la mortalidad neonatal
}

\author{
Diana Isabel Claros Benítez ${ }^{1}$, Luis Alfonso Mendoza Tascón. 1,2 \\ ${ }^{1}$ Facultad Ciencias de la Salud, Programa de Medicina, Unidad Central del Valle. Tuluá, Colombia. ${ }^{2}$ Fundación Hospital \\ San José de Buga. Buga, Colombia.
}

\section{RESUMEN}

Antecedentes: Los trastornos hipertensivos, la diabetes y la obesidad en el embarazo, afectan el peso, la edad gestacional al nacer y la mortalidad neonatal. Objetivo: Evaluar el impacto sobre la mortalidad neonatal, peso al nacer y edad gestacional, de los trastornos hipertensivos (preeclampsia e hipertensión arterial crónica), diabetes (gestacional y no gestacional) y obesidad en la gestación, en un grupo de neonatos nacidos en un hospital colombiano, entre 2005 y 2015. Métodos: Estudio de cohorte retrospectiva, con gestantes con preeclampsia (n: 326), hipertensión arterial crónica (n: 104), diabetes gestacional (n: 246) y obesidad (n: 216). Los datos fueron analizados en Stata® 11.0, empleando mediana o promedio y sus medidas de dispersión, Pruebas t, ranksum, ANOVA o Kruskal Wallis, medidas de frecuencia absolutas y relativas, Pruebas de Chi2 o exacta de Fisher. Para estimar la correlación entre las variables de exposición empleamos el Coeficiente de Correlación de Pearson y para estimar las asociaciones, empleamos riesgo relativo (RR) con su intervalo de confianza de 95\%. La significancia estadística fue definida como un valor $\mathrm{p}<0,05$. Resultados: Hallamos asociación significativa entre bajo peso al nacer y prematuridad con preeclampsia e hipertensión arterial crónica, y mayor peso al nacer con diabetes y obesidad. Hubo también asociación significativa entre mortalidad neonatal e hipertensión arterial crónica y diabetes. Conclusiones: Los trastornos hipertensivos y metabólicos afectan el peso y la edad gestacional al nacer, existiendo asociación significativa entre mortalidad neonatal e hipertensión arterial crónica y diabetes.

\section{PALABRAS CLAVE. Preeclampsia, hipertensión arterial crónica, diabetes, obesidad, mortalidad neonatal}

\section{SUMMARY}

Background: Hypertensive disorders, diabetes and obesity in pregnancy, affect weight, gestational age at birth and neonatal mortality. Objective: Assess the impact on neonatal mortality, birth weight and gestational age, hypertensive disorders (pre-eclampsia and chronic hypertension), diabetes (gestational and non-gestational) and obesity in pregnancy of infants born colombian hospital between, 2005 and 2015. Methods: Retrospective cohort study with pregnant women with preeclapmsia ( $\mathrm{n}$ : 326), chronic hypertension ( $n$ : 104), gestational diabetes ( $n: 246)$ and obesity $(n: 216)$. The data were analyzed in Stata 11.0 using median or average and dispersion measures, t tests, ranksum, ANOVA or Kruskal Wallis, measures absolute and relative frequency, Chi2 tests or Fisher exact. To estimate the correlation between exposure variables we used the Pearson correlation coefficient and to estimate associations employ relative risk (RR) with confidence interval of $95 \%$. Statistical significance was defined as $p<0.05$. Results: We found a significant association between low birth weight and prematurity with preeclampsia and chronic hypertension, and higher birth weight with diabetes and obesity. There was also significant association between neonatal 
mortality and chronic diabetes and hypertension. Conclusions: Hypertensive and metabolic disorders affect weight and gestational age at birth, and there is significant association between neonatal mortality and chronic diabetes and hypertension.

\section{KEY WORDS: Preeclampsia, chronic hypertension, diabetes, obesity, neonatal mortality}

\section{INTRODUCCIÓN}

Los trastornos hipertensivos, la diabetes y la obesidad en el embarazo, afectan la salud de la gestante, el feto y el neonato. Los trastornos hipertensivos del embarazo complican 6-8\% de todos los embarazos, siendo causa importante de mortalidad y morbilidad materna y perinatal en todo el mundo. Se prevé que esta situación empeorará, dada la creciente prevalencia de la obesidad y el síndrome metabólico en las mujeres en edad fértil $(1,2)$. Para las mujeres con diabetes conocida, o para las mujeres que desarrollan la diabetes durante el embarazo, estos cambios pueden poner en riesgo los resultados de la gestación (3). La obesidad puede considerarse como una enfermedad y al mismo tiempo un factor de riesgo de otras enfermedades crónicas. Esta entidad afecta al embarazo a través de otras patologías asociadas, por ejemplo, con la hipertensión arterial y la diabetes (4).

El presente trabajo tuvo por objetivos, evaluar el impacto sobre el peso al nacer, la edad gestacional al nacer y la mortalidad neonatal, en hijos de madres con trastornos hipertensivos (preeclampsia e hipertensión arterial crónica), diabetes (gestacional y no gestacional) y obesidad en la gestación, en un grupo neonatos nacidos en la "Fundación Hospital San José de Buga", Buga, Colombia, entre los años 2005 y 2015.

\section{MATERIALES Y MÉTODOS}

Diseño del estudio: Estudio analítico de cohorte retrospectiva.

Contexto y población de estudio: Trabajo realizado en la ciudad de Buga, con datos recogidos prospectivamente en la "Fundación Hospital San José de Buga", Colombia. La población estuvo constituida por gestantes y sus hijos (neonatos) nacidos en esta institución de salud, entre los años 2005 y 2015.

Criterios de inclusión: Mujeres gestantes de cualquier edad y sus neonatos con edad gestacional $\geq 24$ semanas de gestación y peso al nacer $\geq 500 \mathrm{~g}$, que hayan tenido su parto en la Fundación Hospital San José de Buga, Buga, Colombia entre los años 2005 y 2015. Criterios de exclusión: Mujeres gestantes y sus neonatos con registros incompletos.

Variables dependientes: Peso al nacer en gramos, edad gestacional al nacer en semanas (medido mediante la fecha de última menstruación, ecografía obstétrica más temprana en la gestación, idealmente en el primer trimestre y calificación de Ballard modificado) y mortalidad neonatal. La restricción de crecimiento intrauterino (RCIU) y el neonato grande para la edad gestacional (GEG) fueron clasificados según la curvas de crecimiento intrauterino de Battaglia y Lubchenco (5).

Variables independientes: Edad materna en años cumplidos. Las variables de exposición gestacional fueron preeclampsia (independiente de la gravedad) $(6,7)$, hipertensión arterial crónica (HTAc) diagnosticada preconconcepcional o durante la gestación $(6,8)$, diabetes (gestacional y no gestacional) (9), y obesidad (diagnosticada preconconcepcional o durante la gestación). El grupo no expuesto estuvo constituido por mujeres y sus neonatos que durante la gestación NO hayan estado expuestas a preeclampsia, HTAc, diabetes gestacional y no gestacional y obesidad $(9,10)$.

Fuente de los datos: Base de datos que se lleva en la Fundación Hospital San José de Buga, desde 2005 a 2015 y cuyos objetivos son investigativos.

Método de selección de los participantes: Se incluyeron todos los participantes que cumplieron con los criterios de inclusión y no tenían criterios de exclusión.

Limitaciones y sesgos: Los principales sesgos tenidos en cuenta fueron los de información, controlándose mediante la exclusión de aquellas participantes que no tuvieran la información completa requerida en la investigación y que esta fuera relevante.

Tamaño de la muestra y muestreo: El tamaño de la muestra estuvo constituido por todos los casos de exposición, entre los cuales hubo gestantes con preeclampsia (n: 326), HTAc (n: 104), diabetes gestacional $(92 \%)$ y no gestacional $(8 \%)(n: 246)$ y obesidad (n: 216). Por cada caso de exposición, se escogieron 4 gestantes y sus neonatos no expuestos a preeclampsia, HTAc, diabetes gestacional/no gestacional y obesidad, pareados por la edad gestacional. Las mujeres expuestas fueron incluidas todas si cumplían con los criterios y las no expuestas si cumplían los criterios, mediante muestreo aleatorio simple. Este tamaño de muestra, permitió determinar con el paquete estadístico Epidat $₫$ 3,1, asociaciones estadísticas de riesgo relativos (RR) y Odds Ratio (OR) para cada entidad así: preeclampsia, RR 1,211 y OR 1,421; hipertensión arterial crónica, RR 
1,429 y OR 1,857 ; diabetes gestacional y no gestacional, RR 1,250 y OR 1,5 y obesidad, RR 1,269 y OR 1,538 , con un nivel de confianza de $95 \%$ y poder estadístico de $80 \%$.

Análisis estadístico: Los datos fueron analizados en Stata ${ }^{\circledR}$ 11.0. Para la descripción de las variables categóricas se emplearon frecuencias absolutas y relativas, comparadas mediante la Prueba de Chi2 o exacta de Fisher. Las variables cuantitativas se describieron mediante promedios y su desviación estándar (DE), o medianas y su rango intercuartíl (RI), comparadas mediante Prueba $t$, ranksum, ANOVA o Kruskal Wallis, de acuerdo a su distribución. Para estimar la correlación entre las variables de exposición empleamos el Coeficiente de Correlación de Pearson y para estimar las asociaciones empleamos riesgo relativo (RR) con su intervalo de confianza de 95\% (IC95\%). La significancia estadística fue definida como un valor $\mathrm{p}<0,05$.

Consideraciones éticas: El desarrollo de este estudio se realiza teniendo en cuenta las consideraciones éticas de la Resolución No.008430 del Ministerio de Salud de Colombia, y fue aprobado por los Comité de Ética de la Fundación Hospital San José de Buga y de la Facultad de Ciencias de la Salud de la Unidad Central del Valle del Cauca, Tuluá, Colombia. La investigación carece de conflicto de intereses desde el punto de vista de patrocinios o remuneración alguna.

\section{RESULTADOS}

Un total de 3310 madres y sus hijos fueron incluidos en el estudio. Hubo 326 exposiciones a preeclampsia/eclampsia, 104 a HTAc, 246 gestantes con historia de diabetes gestacional (92\%) y no gestacional (8\%) y 216 a obesidad (Tablas I y II). Aunque la preeclampsia/eclampsia no tuvo un grupo de edad donde fuera significativamente más predominante, la HTAc, la diabetes gestacional/no gestacional y la obesidad, se presentaron con una frecuencia significativamente más elevada entre las madres de $\geq 36$ años de edad (Tabla III).

\section{Tabla I \\ VARIABLES DEMOGRÁFICAS MATERNAS Y NEONATALES}

\begin{tabular}{lc}
\hline Variables & Medida \\
\hline Maternas: & $n: 3310$ \\
Edad (años) & 24 (RI: $10-30)$ \\
Número de embarazo & 2 (RI: $1-3)$ \\
Educación materna & 11 (Rl: $8-11)$ \\
Preeclampsia/eclampsia & $326(10,96 \%)$ \\
Hipertensión arterial crónica & $104(3,78 \%)$ \\
Diabetes gestacional/no & $246(8,50 \%)$ \\
gestacional & $216(7,54 \%)$ \\
Obesidad & \\
Neonatales: & $0(\mathrm{Rl}: 0-0)$ \\
Edad (días) & $38(35-39)$ \\
Edad gestacional (semanas) & $1252(56,29 \%)$ \\
Hombres & $2890(\mathrm{Rl}: 2235-3350)$ \\
Peso al nacer (g) & $1248(56,12 \%)$ \\
Parto vaginal &
\end{tabular}

Tabla II

GRUPOS DE EDAD MATERNA Y EDAD GESTACIONAL NEONATAL

\begin{tabular}{lc}
\hline Grupo de edad & $\mathrm{n}(\%)$ \\
\hline Maternas: & \\
$\leq 19$ años & $848(25,64)$ \\
$20-35$ años & $2130(64,35)$ \\
$\geq 36$ años & $332(10,03)$ \\
Neonatales: & \\
$\leq 27$ semanas & $80(2,42)$ \\
$28-31$ semanas & $230(7,00)$ \\
$32-36$ semanas & $1170(35,35)$ \\
$\geq 37$ semanas & $1830(55,29)$ \\
\hline
\end{tabular}

Tabla III

PREVALENCIA DE EXPOSICIONES SEGÚN GRUPOS DE EDAD MATERNA

\begin{tabular}{|c|c|c|c|c|}
\hline \multirow[t]{2}{*}{ Patologías maternas } & \multicolumn{3}{|c|}{ Grupos de edad } & \multirow[t]{2}{*}{ Valor $\mathrm{p}$} \\
\hline & $\mathrm{n}(\%)$ & $\mathrm{n}(\%)$ & $\mathrm{n}(\%)$ & \\
\hline Preeclampsia/eclampsia & $70(8,25)$ & $223(10,47)$ & $31(9,34)$ & 0,0519 \\
\hline Hipertensión arterial crónica & $2(0,24)$ & $70(3,29)$ & $32(9,64)$ & 0,0001 \\
\hline Diabetes gestacional/gestacional & $26(3,07)$ & $170(7,98)$ & $50(15,06)$ & 0,0001 \\
\hline Obesidad & $36(4,24)$ & $158(7,42)$ & $22(6,63)$ & 0,0019 \\
\hline
\end{tabular}


Hubo una correlación estadísticamente significativa entre obesidad y preeclampsia/eclampsia $(p<0,0142)$, obesidad e HTAc $(p<0,0001)$, obesidad y diabetes gestacional/no gestacional $(p<0,0001)$, diabetes gestacional/no gestacional y preeclampsia $(p<0,0001)$ y diabetes gestacional/no gestacional e HTAc $(p<0,0001)$, no identificándose esta correlación entre HTAc y preeclampsia/eclampsia $(p=0,0592)$.

La obesidad cursó asociada a preeclampsia/ eclampsia en 42 casos $(1,27 \%)$, a HTAc en 28 casos $(0,85 \%)$ y diabetes gestacional/no gestacional en 68 casos (2,05\%). La HTAc se asoció a preeclampsia/eclampsia en 22 casos $(0,66 \%)$ y a diabetes gestacional/no gestacional en 32 casos $(0,97 \%)$. La diabetes gestacional/no gestacional se asoció a preeclampsia/eclampsia en 66 casos (1,99\%).

Los hijos de madres con preeclampsia/eclampsia, tuvieron al nacer $270 \mathrm{~g}$ menos que los no expuestos $(p<0,0001)$, afectando significativamente a neonatos $\geq 28$ semanas. Estos neonatos también al nacer fueron una semana menores que los no expuestos $(p<0,0001)$. A pesar que entre los neonatos hijos de madres con preeclampsia/eclampsia hubo mayor mortalidad, esta diferencia no fue significativa (Tabla IV).

Los hijos de madres con HTAc tuvieron al nacer $390 \mathrm{~g}$ de menos de peso $(p=0,0186)$ y fueron al nacer una semana de gestación menores que los no expuestos $(p=0,0011)$, con una frecuencia significativamente más elevada de mortalidad neonatal $(p<0,0001)$. La HTAc ocasionó una significativa reducción del peso al nacer en los neonatos <28 semanas de gestación, sin afectar significativamente las demás edades gestacionales (Tabla IV).

Los hijos de madres con diabetes gestacional/ no gestacional tuvieron al nacer $460 \mathrm{~g}$ más de peso $(p<0,0001)$, principalmente los neonatos $\geq 32$ y más semanas de gestación, ocasionando una significativa reducción del peso al nacer en los neonatos de 28-31 semanas y no significativa disminución del peso al nacer en los <28 semanas de gestación. En este grupo los neonatos fueron de menor edad gestacional (una semana menos de edad gestacional; $p<0,0001)$, con una significativa mayor mortalidad ( $p<0,0430)$ (Tabla IV).

Los hijos de madres obesas fueron significativamente de mayor peso al nacer (640 $\mathrm{g}$ de peso más de peso al nacer; $p<0,0001)$, principalmente los $\geq 32$ y más semanas de gestación, ocasionando una no significativa reducción del peso al nacer en los neonatos de 28-31 semanas y en los <28 se- manas de gestación. Estos neonatos tuvieron una edad gestacional mayor al nacer (una semana más de edad gestacional; $p<0,0001)$, sin afectar significativamente la mortalidad neonatal (Tabla IV).

Hallamos una asociación significativa entre los trastornos hipertensivos (preeclampsia/eclampsia e hipertensión arterial crónica) con bajo peso al nacer (<2500 g) y prematuridad (<37 semanas de gestación), mientras la diabetes gestacional/no gestacional y la obesidad, se asociaron con un peso al nacer $>3200 \mathrm{~g}$ y >3999 g. Encontramos una asociación significativa entre RCIU y preeclampsia/eclampsia (RR: 1,87; IC95\%: 1,53-2,28; p<0,001) e HTAc (RR: 2,50; IC95\%: 1,91-3,27; $p<0,001)$, y peso grande para la edad gestacional con diabetes gestacional y no gestacional (RR: 5,70; IC95\%: 4,31-7,54; $p<0,001$ ) y obesidad (RR: 5,23; IC95\%: 3,88-7,08; $p<0,001$ ). Hallamos asociación significativa entre nacer con peso $\geq 4500$ g y diabetes gestacional/no gestacional (RR: 32,29; IC95\%: 10,49-99,37; $p<0,001)$ y obesidad (RR: 24,52; IC95\%: 7,44-80,77; p<0,001).

La mortalidad neonatal se asoció significativamente a HTAc y diabetes gestacional/no gestacional, y aunque fue más elevada en neonatos de madres con preeclampsia/eclampsia y obesidad, no se halló una asociación estadísticamente significativa (Tabla V). Igualmente, se halló una asociación significativa entre mortalidad neonatal y bajo peso al nacer (<2500 $\mathrm{g}$ de peso al nacer) con RR: 2,26 (IC95\%: 2,06-2,47; $p<0,0001)$ y prematuridad (<37 semanas de gestación) con RR: 2,10 (IC95\%: 1,95$2,27 ; p<0,0001)$. Estas muertes ocurrieron en las primeras 24 horas en 29 neonatos (31,52\%), entre el 2-7 días 42 neonatos (45,65\%) y entre el 8-28 día 21 neonatos $(22,82 \%)$.

\section{DISCUSIÓN}

Este trabajo evaluó el impacto sobre el peso, edad gestacional al nacer y mortalidad neonatal, de la preeclampsia/eclampsia, HTAc, diabetes gestacional/no gestacional y obesidad.

En este grupo de gestantes, el principal evento fue la preeclampsia/eclampsia, seguido por diabetes, obesidad e HTAc. La hipertensión inducida por el embarazo afecta a $10 \%$ de éstas y la preeclampsia complica a $2-8 \%$ de embarazos (11). La eclampsia ocurre en 1/2000 gestaciones en países de altos ingresos (12) y 1/100-1/1700 en países de pobre desarrollo $(13,14)$. Se estima que alrededor de 5/1000 gestantes en la Gran Bretaña padecen preeclampsia grave $(12,15)$. 


\section{Tabla IV}

\section{PESO AL NACER, EDAD GESTACIONAL AL NACER Y MORTALIDAD NEONATAL, EN MADRES CON HISTORIA DE PREECLAMPSIA/ECLAMPSIA, HIPERTENSIÓN ARTERIAL CRÓNICA, DIABETES GESTACIONAL/NO GESTACIONAL Y OBESIDAD}

\begin{tabular}{|c|c|c|c|}
\hline $\begin{array}{l}\text { Variables } \\
\text { resultado }\end{array}$ & $\begin{array}{l}\text { No expuestas } \\
\text { n: } 2648\end{array}$ & $\begin{array}{c}\text { Preeclampsia/eclampsia } \\
\text { n: } 326\end{array}$ & Valor $p$ \\
\hline Peso al nacer (g) & 2740 (RI: 2135-3220) & 2460 (RI: 1800-3070) & $<0,0001$ \\
\hline$E G<28$ semanas & 1050 (RI: 920-1130) & 1040 (RI: 1020-1040) & 0,7756 \\
\hline EG 28-31 semanas & 1440 (RI: 1223-1655) & 1260 (RI: 1060-1460) & 0,0071 \\
\hline EG 32-36 semanas & 2205 (RI: 1960-2530) & 2003 (RI: 1790-2660) & 0,0027 \\
\hline$E G \geq 37$ semanas & 3190 (RI: 2815-3460) & 3050 (RI: 2690-3470) & 0,0394 \\
\hline EG (semanas) & 37 (RI: 34-39) & 36 (RI: 34-38) & $<0,0001$ \\
\hline Muerte & $70(2,89 \%)$ & $6(1,84 \%)$ & 0,3860 \\
\hline $\begin{array}{l}\text { Variables } \\
\text { resultado }\end{array}$ & $\begin{array}{l}\text { No expuestas } \\
\text { n: } 2648\end{array}$ & $\begin{array}{c}\text { Hipertensa } \\
\text { arterial crónica } \\
\text { n:104 }\end{array}$ & Valor $\mathrm{p}$ \\
\hline Peso al nacer (g) & 2740 (RI: 2135-3220) & 2350 (RI: 2003-2940) & 0,0186 \\
\hline$E G<28$ semanas & 1050 (RI: 920-1130) & 720 (RI: 720-720) & 0,0201 \\
\hline EG 28-31 semanas & 1440 (RI: 1223-1655) & 1313 (RI: 1065-1610) & 0,1787 \\
\hline EG 32-36 semanas & 2205 (RI: 1960-2530) & 2220 (RI: 2040-2650) & 0,5148 \\
\hline$E G \geq 37$ semanas & 3190 (RI: 2815-3460) & 2905 (RI: 2550-3520) & 0,1843 \\
\hline EG (semanal) & 37 (RI: 34-39) & 36 (RI:34-38) & 0,0011 \\
\hline Muerte & $30(1,24 \%)$ & $14(13,46 \%)$ & $<0,0001$ \\
\hline $\begin{array}{l}\text { Variables } \\
\text { resultado }\end{array}$ & $\begin{array}{l}\text { No expuestas } \\
\text { n: } 2648\end{array}$ & $\begin{array}{c}\text { Diabetes gestacional/ } \\
\text { no gestacional } \\
\text { n: } 246\end{array}$ & Valor $\mathrm{p}$ \\
\hline Peso al nacer (G) & 2740 (RI: 2135-3220) & 3200 (RI: 2610- 3750) & $<0,0001$ \\
\hline$E G<28$ semanas & 1050 (Rl: 920-1130) & 960 (RI: 960-960) & 0,1178 \\
\hline EG 28-31 semanas & 1440 (RI: 1223-1655) & 1065 (RI: 1063-2243) & 0,0158 \\
\hline EG 32-36 semanas & 2205 (RI: 1960-2530) & 2650 (RI: 2100-2940) & $<0,0001$ \\
\hline$E G \geq 37$ semanas & 3190 (RI: 2815-3460) & 3540 (RI: 3170-3820) & $<0,0001$ \\
\hline EG (semanal) & 38 (RI: 35-39) & 37 (RI: 35-39) & $<0,0001$ \\
\hline Muerte & $70(2,89 \%)$ & $12(4,88 \%)$ & 0,0430 \\
\hline $\begin{array}{l}\text { Variables } \\
\text { resultado }\end{array}$ & $\begin{array}{l}\text { No expuestas } \\
\text { n: } 2648\end{array}$ & $\begin{array}{l}\text { Obesidad } \\
\mathrm{n}: 216\end{array}$ & Valor $\mathrm{p}$ \\
\hline Peso al nacer (G) & 2740 (RI: 2135-3220) & 3380 (RI: 2680-3700) & $<0,0001$ \\
\hline$E G<28$ semanas & 1050 (RI: 920-1130) & 960 (RI: 960-960) & 0,1172 \\
\hline EG 28-31 semanas & 1440 (RI: 1223-1655) & 1108 (RI: 1065-3325) & 0,2310 \\
\hline EG 32-36 semanas & 2205 (RI: 1960-2530) & 2480 (RI: 2060-3440) & 0,0048 \\
\hline$E G \geq 37$ semanas & 3190 (RI: 2815-3460) & 3575 (RI: 3260-3820) & $<0,0001$ \\
\hline EG (semanal) & 37 (RI: 34-39) & 38 (RI: 35.5- 39) & $<0,0001$ \\
\hline Muerte & $70(2,89 \%)$ & $10(4,63 \%)$ & 0,0890 \\
\hline
\end{tabular}

No expuestas: Madres que no estuvieron expuestas en esta gestación a preeclampsia/eclampsia, hipertensión arterial crónica, diabetes gestacional/no gestacional y obesidad. 


\section{Tabla V \\ ASOCIACIÓN ENTRE PREECLAMPSIA/ECLAMPSIA, HIPERTENSIÓN ARTERIAL CRÓNICA, DIABETES GESTACIONAL Y NO GESTACIONAL, OBESIDAD Y PESO AL NACER, EDAD GESTACIONAL AL NACER Y MORTALIDAD NEONATAL}

\begin{tabular}{|c|c|c|c|c|}
\hline $\begin{array}{l}\text { Variables } \\
\text { resultado }\end{array}$ & $\begin{array}{l}\text { No expuestas } \\
\mathrm{n}: 2648\end{array}$ & $\begin{array}{c}\text { Preeclampsia / eclampsia } \\
\text { n: } 326\end{array}$ & $\mathrm{RR}(\mathrm{IC} 95 \%)$ & Valor $p$ \\
\hline Peso al nacer $<2500 \mathrm{~g}$ & $1064(40,00 \%)$ & $168(51,53 \%)$ & $1,50(1,22-1,84)$ & 0,0001 \\
\hline Edad gestacional $<37$ s & $1184(48,97 \%)$ & $188(13,7 \%)$ & $1,59(1,29-1,96)$ & $<0,0001$ \\
\hline Muerte & $70(2,89 \%)$ & $6(7,89 \%)$ & $0,71(0,33-1,55)$ & 0,3860 \\
\hline $\begin{array}{l}\text { Variables } \\
\text { resultado }\end{array}$ & $\begin{array}{c}\text { No expuestas } \\
\text { n: } 2648\end{array}$ & $\begin{array}{c}\text { Hipertensa } \\
\text { arterial crónica } \\
\text { n:104 }\end{array}$ & RR (IC 95\%) & Valor $\mathrm{p}$ \\
\hline Peso al nacer $<2500 \mathrm{~g}$ & $1064(40,00 \%)$ & $58(5,16 \%)$ & $1,83(1,25-2,67)$ & 0,0015 \\
\hline Edad gestacional $<37 \mathrm{~s}$ & $1184(48,97 \%)$ & $64(5,12 \%)$ & $1,93(1,31-2,84)$ & 0,0007 \\
\hline Muerte & $70(2,89 \%)$ & $14(16,66 \%)$ & $4,94(2,94-8,31)$ & $<0,0001$ \\
\hline $\begin{array}{l}\text { Variables } \\
\text { resultado }\end{array}$ & $\begin{array}{c}\text { No expuestas } \\
\text { n: } 2648\end{array}$ & $\begin{array}{c}\text { Diabetes gestacional/ } \\
\text { no gestacional } \\
\text { n: } 246\end{array}$ & RR (IC 95\%) & Valor $p$ \\
\hline Peso al nacer $>3200 \mathrm{~g}$ & $690(28,54 \%)$ & $122(15,02 \%)$ & $2,52(1,99-3,20)$ & $<0,0001$ \\
\hline Peso al nacer $>3999 \mathrm{~g}$ & $52(2,15 \%)$ & $20(27,8 \%)$ & $3,47(2,34-5,14)$ & $<0,0001$ \\
\hline Edad gestacional al nacer $<37 \mathrm{~s}$ & $1184(48,97 \%)$ & $106(8,21 \%)$ & $0,94(0,34-1,20)$ & 0,6241 \\
\hline Muerte & $70(2,89 \%)$ & $12(14,63 \%)$ & $1,76(1,03-3,01)$ & 0,0433 \\
\hline $\begin{array}{l}\text { Variables } \\
\text { resultado }\end{array}$ & $\begin{array}{c}\text { No expuestas } \\
\text { n: } 2648\end{array}$ & $\begin{array}{c}\text { Obesidad } \\
n: 216\end{array}$ & RR (IC 95\%) & Valor $p$ \\
\hline Peso al nacer $>3200 \mathrm{~g}$ & $690(28,54 \%)$ & $132(16,06 \%)$ & $3,90(3,01-5,07)$ & $<0,0001$ \\
\hline Peso al nacer $>3999 \mathrm{~g}$ & $52(2,15 \%)$ & $26(33,33 \%)$ & $4,89(3,47-6,88)$ & $<0,0001$ \\
\hline Edad gestacional al nacer $<37 \mathrm{~s}$ & $1184(48,97 \%)$ & $68(5,43 \%)$ & $0,59(0,45-0,78)$ & 0,0002 \\
\hline Muerte & $70(2,89 \%)$ & $10(12,5 \%)$ & $1,68(0,93-3,06)$ & 0,0885 \\
\hline
\end{tabular}

No expuestas: Madres que no estuvieron expuestas en esta gestación a preeclampsia/eclampsia, hipertensión arterial crónica, diabetes gestacional/no gestacional y obesidad.

La diabetes pregestacional/gestacional y la obesidad, son entidades relativamente frecuentes en la gestación. Un 5\% de estas mujeres que tienen partos, presentan diabetes preexistente 0 diabetes gestacional. Se estima entre estas, que $87,5 \%$ tienen diabetes gestacional, $7,5 \%$ diabetes tipo 1 y $5 \%$ tipo 2. (16). Para el año 2015, la Organización Mundial de la Salud estimó una mayor epidemia de sobrepeso (2,3 billones de adultos $\geq 15$ años) y obesidad ( \pm 700 millones de adultos), más pronunciado en personas jóvenes, incluyendo mujeres en edad reproductiva, la cual ocurre tanto antes, como durante la gestación, con ganancia de peso excesiva (17). Para países de América Latina como México, la prevalencia de obesidad alcanzó
$10-20 \%$ en infantes, $30-40 \%$ en adolescentes y $60-$ $70 \%$ en adultos $(18,19)$. En países desarrollados como Estados Unidos de América (EUA), más de $30 \%$ de mujeres son obesas, más de $50 \%$ de gestantes tienen sobrepeso y $8 \%$ de mujeres en edad reproductiva tienen obesidad mórbida. La prevalencia de obesidad en el embarazo tiene rangos de 11$22 \%$ (20). En este país, la prevalencia de obesidad en mujeres de 20-39 años pasó de 28,4\% en 1999 a $34 \%$ en 2008, mientras que para Polonia fue $25,6 \%$, Francia $27,2 \%$ y Eslovenia $27,8 \%$. La mayoría de los países europeos tienen prevalencias de obesidad que oscilan entre $30-37 \%$, mientras Escocia tiene una prevalencia elevada de $48,4 \%$ (21). 
Los trastornos hipertensivos del embarazo son entidades comunes, complicando $6-8 \%$ de todos los embarazos $(22,23)$. La prevalencia de HTAc en los EUA está estimada en 3\% (8). En Inglaterra se estima que la HTAc complica a $12-15 \%$ de todos los embarazos (6).

Este trabajo identificó una correlación significativa entre las diferentes patologías evaluadas, excepto entre HTAc y preeclampsia/eclampsia. La HTAc, la diabetes y la obesidad afectaron principalmente a gestantes $\geq 36$ años. La prevalencia de HTAc en EUA ha aumentado, asociándose a otros trastornos como la obesidad (8). La incidencia de la diabetes gestacional también está aumentando como resultado de mayores tasas de obesidad en la población general y más embarazos en mujeres de edad avanzada (16). La obesidad se ha asociado a HTAc (aumento de riesgo 3-10 veces), preeclampsia (aumento de riesgo 2-3 veces), diabetes pregestacional (aumento de riesgo 3-10 veces) y gestacional (17). Aunque en este trabajo no hallamos correlación entre hipertensión arterial crónica y preeclampsia/eclampsia, Seely y cols (24) describen que en mujeres con HTAc, hay un aumento de la frecuencia de preeclamsia (17 a $25 \%$ vs 3 a $5 \%$ comparado con la población general).

En cuanto la asociación entre obesidad y diabetes, la literatura informa esta asociación, encontrándose que las mujeres con obesidad tienen 3,05-4,21 veces el riesgo de desarrollar diabetes gestacional y con obesidad grave (IMC >33 a >40 $\mathrm{kg} / \mathrm{m} 2$ ) 5,07-16,04 veces el riesgo de hacer diabetes gestacional, comparado con mujeres de peso normal. Las mujeres obesas también tienen un riesgo aumentado de presentar preeclampsia. Se ha encontrado que mujeres con IMC de $30-34,9 \mathrm{~kg} /$ $\mathrm{m} 2$ y obesidad grave $\geq 35 \mathrm{~kg} / \mathrm{m} 2$, tienen riesgos relativos agrupados de 2,68 (IC95\%: 2,40-3,00) y 3,43 (IC95\%: 2,59-4,51) respectivamente. Otro trabajo halló que las mujeres obesas tienen de 3-10 veces mayor probabilidad de desarrollar preeclampsia $(17,21)$.

Identificamos que los trastornos hipertensivos que afectan el embarazo, afectan el peso al nacer, la edad gestacional al nacer y la mortalidad neonatal. Encontramos que los hijos de madres con preeclampsia/eclampsia, tuvieron al nacer un significativo menor peso y menor edad gestacional, existiendo una asociación significativa entre estas y RCIU y prematuridad, pero no hallada con mortalidad neonatal. Salinas y cols (25), encontraron que la preeclampsia se halló asociado a bajo peso al nacer y a prematuridad. Este hallazgo es similar al de Groom y cols (26) para quienes la preeclampsia fue riesgo de bajo peso para la edad gestacional en neonatos <34 semanas y entre los de 34-36 semanas principalmente. La preeclampsia se ha asociado fuertemente a bajo peso al nacer, dado que está asociada a prematuridad, pero también por las afectaciones placentarias ocurridas, que conducen a disfunción de esta, con reducción de flujo sanguíneo placentario que ocasiona hipoxia placentaria, reducción de la nutrición fetal, con subsecuente RCIU (27-29). Los informes sobre muertes perinatales muestran que $5 \%$ de los nacidos muertos sin anomalías congénitas, ocurrieron en madres con preeclampsia (30). La hipertensión en el embarazo se asocia con $8-10 \%$ de todos los nacimientos prematuros y más del $50 \%$ de mujeres con preeclampsia grave tienen partos prematuros (30). La HTAc se asocia a RCIU complicando $10-20 \%$ de embarazos $(8,31,32,33)$. El análisis del Danish National Birth Cohort, informa que la HTAc después de ajustar por edad, índice de masa corporal, consumo de tabaco, paridad y diabetes, aumenta el riesgo de parto prematuro en cinco veces y aumenta en un $50 \%$ el riesgo de nacer con bajo peso para la edad gestacional (34). Tal como nuestros hallazgos, la HTAc, también aumenta el riesgo de mortalidad neonatal (8).

Los hijos de madres con diabetes y obesidad, tuvieron un significativo mayor peso al nacer, que los no expuestos. No hubo asociación entre estas dos entidades y prematuridad, pero si se halló asociación significativa entre diabetes y mortalidad neonatal. Los hijos de madres con diabetes y obesidad, tuvieron un riesgo aumentado de nacer con peso superior a $3200 \mathrm{~g}$ y $3999 \mathrm{~g}$. El feto hijo de madre con diabetes está en riesgo de padecer macrosomía o RCIU. El riesgo de macrosomía ocurre con mayor frecuencia cuando existe un mal control de la glicemia, mientras la RCIU es mayor en mujeres con complicaciones vasculares (retinopatía o nefropatía) o cuando desarrollan preeclampsia $(35,36)$. La macrosomía fetal, se ha asociado a mortalidad intrauterina, traumas al nacer, mortalidad perinatal y postnatal (36). La obesidad también aumenta el riesgo de macrosomía fetal (OR 1,4; IC95\%: 1,21,7 ) y de muerte perinatal (OR 2,79; IC95\%: 1,94$4,02)$ de causa no conocida, quizás asociado a comorbilidades como los trastornos hipertensivos (10). Owens y cols (37) describen que la macrosomía se presentó en $15,5 \%, 21,4 \%$ y $27,8 \%$ de la mujeres con peso normal, sobrepeso y obesidad respectivamente $(p<0,01)$. Las distocias de hombro se presentaron en $4 \%$ (neonatos con peso al nacer $>4 \mathrm{~kg}$ ), comparado con $0,2 \%$ (neonatos con peso al nacer $<4 \mathrm{~kg})(\mathrm{p}<0,01)$. Para Marchi y cols (21) el riesgo de nacer con un peso grande para la edad gestacional $(>4.000 \mathrm{~g})$ se aumentó en un 100\% (OR: 2,00; IC95\%:1,84-2,18) y de macrosomía (>4.500 g) se aumentó en un 223\% (OR: 3,23; IC95\%: 2,39-4,37).

La obesidad aumenta el riesgo de parto prematuro. El riesgo de nacer $<32$ semanas de gestación en mujeres con índice de masa corporal $>40 \mathrm{~kg} / \mathrm{m} 2$ es alto (ORa: 2,27; IC95\%: 1,76-2,94). Para gestantes con índice de masa corporal $\geq 35 \mathrm{~kg} / \mathrm{m} 2$, el 
riesgo de prematuridad se eleva en un 33\% (ORa: 1,33; IC95\%: 1,12-1,57) (21). La mortalidad neonatal se halla aumentada en mujeres con índice de masa corporal $\geq 30 \mathrm{~kg} / \mathrm{m} 2$, $\geq 35 \mathrm{~kg} / \mathrm{m} 2$ y $\geq 40 \mathrm{~kg} /$ m2 (RR: 1,34; IC95\%: 1,22-1,47; RR: 1,97; IC95\%: 1,71-2,28 y RR: 3,54; IC95\%: 2,56-4,89, respectivamente). El riesgo de abortos también se encuentra aumentado (RR: 1,31; IC95\%: 1,18-1,43), mientras que el riesgo de mortinato se encuentra aumentado en un 46\% (RR: 1,46; IC95\%: 1,37-1,55) (21).

\section{CONCLUSIONES}

Los trastornos hipertensivos y los trastornos metabólicos (diabetes y obesidad), se encuentran directamente asociados a resultados fetales y neonatales, específicamente peso al nacer, edad gestacional al nacer y mortalidad neonatal. Encontramos que los hijos de madres con preeclampsia/ eclampsia o HTAc nacen con un peso significativamente menor, afectando la primera entidad a los de $>28$ semanas de edad gestacional y la segunda a $<28$ semanas de edad gestacional. De estas dos entidades, encontramos que la HTAc se halló asociada a mortalidad neonatal. Por su parte, la diabetes y la obesidad, también están afectando el peso fetal y por ende al nacer; los hijos de madres con estas dos entidades tienen pesos mucho mayores que los no expuestos. A pesar que entre los hijos de madres obesas y con diabetes hubo mayor mortalidad, sólo se logró demostrar asociación significativa con diabetes. La situación es más grave aún, dado que dos o más de estas entidades pueden coexistir en una gestación, agravando los resultados, tanto para la gestante, como para el feto y el neonato.

Se debe identificar a las mujeres en riesgo de preeclampsia y diabetes gestacional, como también a las mujeres con HTAc, diabetes preconcepcional y obesidad previa al embarazo, lo cual permitirá realizar intervenciones precoces y tratamientos oportunos, con lo cual se reduciría el riesgo de morbi-mortalidad materno-perinatal.

\section{REFERENCIAS}

1. National High Blood Pressure Education Program. Report of the National High Blood Pressure Education Program Working Group on High Blood Pressure in Pregnancy. Am J Obstet Gynecol 2000;183:1-22.

2. Yoder SR, Thornburg LL, Bisognano JD. Hypertension in pregnancy and women of childbearing age. Am J Med 2009;122:890-5.

3. IDF Clinical Guidelines Task Force. Global Guideline on Pregnancy and Diabetes. Brussels: International Diabetes Federation, 2009. Acceso: 21-2-2016. Disponible en: https://www.idf.org/webdata/docs/Pregnancy_EN_RTP.pdf.

4. González J, Juárez JS, Rodríguez J. Obesidad y embarazo. Revista Médica MD 2013;4(4):269-75.
Acceso: 23-2-2016. Disponible en: http://www.revistamedicamd.com/sites/default/files/revistas/art_rev_-_ obesidad_y_embarazo_rev_med_md_2013_44.pdf.

5. Battaglia FC, Lubchenco LO. A practical classification of newborn infants by weight and gestational age. $\mathrm{J}$ Pediatr 1967;71(2):159-63.

6. NICE National Institute for Health and Care Excellence. NICE support for commissioning for hypertension in pregnancy. 2013. Acceso: 21-02-2016. Disponible en: http://www.nice.org.uk/guidance/qs35/resources/ support-for-commissioning-for-hypertension-in-pregnancy-253628461.

7. NICE Clinical Guideline: Hypertension in pregnancy: the management of hypertensive disorders during pregnancy. 2010. Acceso: 21-02-2016. Disponible en: http://www.nice.org.uk/guidance/qs35/resources/ support-for-commissioning-for-hypertension-in-pregnancy-253628461.

8. Sibai BM. Chronic hypertension in pregnancy. Obstet Gynecol 2002;100:369-77.

9. IDF Clinical Guidelines Task Force. Global Guideline on Pregnancy and Diabetes. Brussels: International Diabetes Federation, 2009. Acceso: 21-2-2016. Disponible en: https://www.idf.org/webdata/docs/Pregnancy_EN_RTP.pdf.

10. Davies G, Maxwell C, McLeod L, and Maternal Fetal Medicine Committee. Obesity in pregnancy. J Obstet Gynaecol Can 2010;32(2):165-73.

11. WHO International Collaborative Study of Hypertensive Disorders of Pregnancy. Geographic variation in the incidence of hypertension in pregnancy. Am J Obstet Gynecol 1988;158:80-3.

12. Douglas K, Redman C. Eclampsia in the United Kingdom. BMJ 1994;309:1395-400.

13. Crowther CA. Eclampsia at Harare maternity hospital. An epidemiological study. S Afr Med J 1985;68:927-9.

14. Bergstrom S, Povey G, Songane F, et al. Seasonal incidence of eclampsia and its relationship to meteorological data in Mozambique. J Perinat Med 1992;20:153-8.

15. Tuffnell DJ, Jankowicz D, Lindow SW, Lyons G, Mason GC, Russell IF, Walker JJ. Outcomes of severe pre-eclampsia/eclampsia in Yorkshire 1999/2003. BJOG 2005; 112:875-80.

16. NICE National Institute for Health and Care Excellence Diabetes in pregnancy: management from preconception to the postnatal period. NICE guideline Published: 25 February 2015 Acceso: 23-2-2016. Disponible en: http://www.nice.org.uk/guidance/ng3/ resources/diabetes-in-pregnancy-management-frompreconception-to-the-postnatal-period-51038446021.

17. Saskatchewan Prevention Institute. Maternal obesity excessive gestational weight gain and pregnancy outcomes. Mayo 2010. Acceso: 16-03-2016. Disponible: http://www.skprevention.ca/wp-content/ uploads/2013/01/Maternal_Obesity_Excessive_Gestational_Weight_Gain.pdf.

18. Reaven G. Metabolic syndrome: Pathophysiology and implications for management of cardiovascular disease. Circulation 2002;106:286-8.

19. ENSANUT 2012. Encuesta Nacional de Salud y Nutrición 2012. Resultados nacionales. Primera edición. 2012. Disponible en: http://ensanut.insp.mx/.

20. Heslehurst N, Ellis LJ, Simpson H. Trends in maternal obesity incidence, rates, demographic predictors, and 
health inequalities in 36,821 women over a 15 years period. Br J Obstet Gynecol 2007; 114:187-94.

21. Marchi J, Berg M, Dencker A, Olander E.K, Begley C. Risk associated with obesity in pregnancy, for the mother and baby: a systematic review of reviews. Obesity Rev 2015;16:621-38.

22. National High Blood Pressure Education Program (2000) Report of the National High Blood Pressure Education Program Working Group on High Blood Pressure in Pregnancy. Am J Obstet Gynecol 183:122.

23. Yoder SR, Thornburg LL, Bisognano JD. Hypertension in pregnancy and women of childbearing age. Am J Med 2009122:890-5.

24. Seely E, Ecker J. Chronic hypertension in pregnancy. N Engl J Med 2011;365:439-46.

25. Salinas H, Erazo M, Pastene C, Reyes A, Catalán J, Carmona S, et al. Factores de riesgo asociados al bajo peso al nacer. Rev Chil Salud Pública 2004;8(2):7883.

26. Groom KM, North RA, Poppe KK, Sadler L, McCowan LME. The association between customized small for gestational age infants and pre-eclampsia or gestational hypertension varies with gestational at delivery. BJGO 2007; 114:478-84.

27. Rasmussen S, Irgens LM. Fetal growth and body proportion in preeclampsia. Obstet Gynecol 2003;101:575-83.

28. Xiao R, Sorensen TK, Williams MA, Ujthy DA. Influence of pre-eclampsia on fetal growth. Matern Fetal Neonatal Med 2003;13:157-62.

29. Vatten LJ, Skjaerven R. Is pre-eclampsia more than one disease? BJOG 2004;111:298-302.
30. NICE National Institute for Health and Care Excellence. Hypertension in pregnancy. Published date: July 2013. Acceso: 21-02-2016. Disponible en: http://www. nice.org.uk/guidance/QS35/chapter/Introduction.

31. Sibai BM, Lindheimer M, Hauth J, et al. Risk factors for preeclampsia, abruptio placentae, and adverse neonatal outcomes among women with chronic hypertension. N Engl J Med 1998;339:667-71.

32. Rey E, Couturier A. The prognosis of pregnancy in women with chronic hypertension. Am J Obstet Gynecol 1994;171:410-6.

33. McCowan LM, Buist RG, North RA, Gamble G. Perinatal morbidity in chronic hypertension. $\mathrm{Br} \mathrm{J}$ Obstet Gynaecol 1996;103:123-9

34. Ananth CV, Peltier MR, Kinzler WL, Smulian JC, Vintzileos AM. Chronic hypertension and risk of placental abruption: is the association modified by ischemic placental disease? Am J Obstet Gynecol 2007;197:273. el-273.e7.

35. NHS Quality Improvement Scotland (NHS QIS). Management of diabetes. A national clinical guideline. March 2010 Updated September 2013. Acceso: 232-2016. Disponible en: http://www.sign.ac.uk/pdf/ sign116.pdf.

36. NICE National Institute for Health and Care Excellence Diabetes in pregnancy: management from preconception to the postnatal period. NICE guideline Published: 25 February 2015 Acceso: 23-2-2016. Disponible en: http://www.nice.org.uk/guidance/ng3/ resources/diabetes-in-pregnancy-management-frompreconception-to-the-postnatal-period-51038446021.

37. Owens LA, O"Sullivan EP, Kirwan B, Avalos G, Gaffney G, Dunne F. ATLANTIC DIP: The Impact of obesity on pregnancy outcome in glucose-tolerant women. Diabetes Care 2010;33:577-9. 